\title{
Location of the elevation axis in a large optical telescope
}

\author{
Stephen Padin
}

\begin{abstract}
Proposed designs for the next generation of large optical telescopes favor a tripod or quadrupod secondary support, and a primary supported from the back, but it is not yet clear whether the elevation axis should be in front of the primary or behind it. A study is described of the effect of elevation-axis location on key performance parameters (fundamental frequency, blockage, and wind-induced secondary decenter) for a 30-m Cassegrain telescope with a mount configuration that is typical of the new designs. For a fast (e.g., $f / 1$ ) primary, the best location for the elevation axis is behind the primary. The penalty for moving the elevation axis in front of the primary is roughly a $40 \%$ decrease in fundamental frequency and a corresponding reduction in the control bandwidth for pointing and optical alignment. (c) 2004 Optical Society of America
\end{abstract}

OCIS codes: $350.1260,220.4880$.

\section{Introduction}

The position of the elevation axis in a large telescope is an important design consideration because it affects many key parameters, e.g., the fundamental frequency of the structure, the blockage that is due to the secondary, the wind-induced decenter of the secondary, the size and cost of the telescope enclosure, and the location of instruments. There are many optical and radio telescope designs, with a wide range of elevation axis locations, but there are no quantitative studies of the effect of moving the elevation axis in a particular design. Such a study is the goal in this paper. The analysis is done first for a conventional optical telescope with a tube on a fork mount. This is a familiar configuration, with many examples that can provide at least a qualitative check of the results. The emphasis of the paper is on a quadrupod and c-ring mount configuration, which is typical of the new designs for large optical telescopes (and many existing radio telescopes).

Most optical telescopes have a tube structure with a spider to support the secondary mirror (or prime focus instrument). This configuration has low support blockage, typically just $1-2 \%$, but the

The author (spadin@caltech.edu) is with the California Institute of Technology, MS 105-24, Pasadena, California 91125.

Received 8 May 2003; revised manuscript received 20 October 2003; accepted 22 October 2003.

0003-6935/04/051097-11\$15.00/0

(C) 2004 Optical Society of America wind cross section of the upper tube and spider is quite large (see Section 2 below). In most telescopes (e.g., Keck, ${ }^{1}$ the Very Large Telescope, ${ }^{2}$ Subaru, ${ }^{3}$ and Magellan ${ }^{4}$ ), the elevation axis, which provides access to the Nasmyth foci, is in front of the primary. (An exception is Gemini, ${ }^{5}$ which cannot support a Nasmyth focus because the elevation axis intersects the primary.) Proposed designs for the next generation of large optical telescopes have abandoned the tube, or at least its upper section, in favor of a tripod or quadrupod secondary support to reduce the effects of wind buffeting (see Section 4 below). Most of the designs [e.g., the Giant Segmented Mirror Telescope ${ }^{6}$ and Euro50 (Ref. 7)] have the elevation axis behind the primary, as in a typical radio telescope; the California Extremely Large Telescope $^{8}$ (CELT) has its elevation axis in front, so multiple Nasmyth instruments can be illuminated simply by tilting the tertiary.

Moving the elevation axis toward the secondary decreases the size of the secondary required for an instrument at the Nasmyth focus, but the mass of the secondary support must be increased to balance the telescope, thus decreasing the fundamental frequency of the structure. A more massive or a shorter secondary support is stiffer, so moving the elevation axis closer to the secondary also reduces the wind-induced decenter of the secondary. The tradeoff between these effects is explored in the following sections with simple, lumped-element, coupledoscillator structural models applied to a $30-\mathrm{m}$ Casse- 


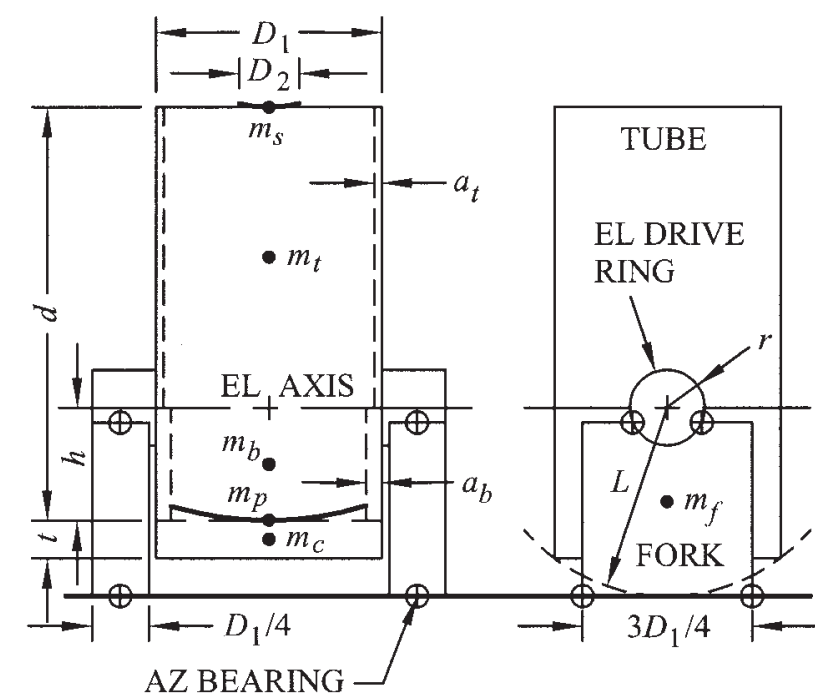

Fig. 1. Telescope with a tube and a fork mount. Filled circles, centers of mass of the telescope components.

grain telescope with a flat tertiary feeding the Nasmyth focus.

\section{Telescope with a Tube on a Fork Mount}

\section{A. Structure}

Figure 1 shows a telescope with a conventional tubestyle secondary support and a simple fork mount (roughly the same size as the arms of the Keck yoke structure $^{1}$ ). The elevation (EL) bearings support rings attached to the tube, and the azimuth (AZ) bearings run on a track on a stiff pier. If the pier is not stiff, it will participate in the fundamental mode of the telescope, giving a lower fundamental frequency. All the structures in Fig. 1 are drawn as solids, but in practice they would be space frames. Typical design constraints for existing telescopes of this type are that they have (1) a balanced tube and (2) matched gravitational deflections in the top and bottom tubes to maintain alignment of the optics as the telescope elevation changes. The second constraint is one of several options, but it is a common approach. If the secondary is equipped with active position control, we might instead design the tube for minimum mass, to give a high fundamental frequency, or for a particular stiffness, to limit the windinduced decenter of the secondary. In the following analysis, all the structures are made from steel, which reduces the cost, but it may be practical to make the top tube from a composite. This would reduce the tube mass and increase the fundamental frequency of the telescope.

Refer to Fig. 1; for a balanced tube,

$$
m_{s}(d-h)+m_{t} \frac{(d-h)}{2}=m_{b} \frac{h}{2}+m_{p} h+m_{c}\left(h+\frac{t}{2}\right),
$$

where $m_{s}$ is the mass of the secondary $\left(\sim 10^{4} \mathrm{~kg}\right.$ for a 4-m-diameter secondary ${ }^{8}$ ),

$$
m_{t}=\pi D_{1} a_{t}(d-h) \eta_{t} \rho
$$

is the mass of the top tube,

$$
m_{b}=\pi D_{1} a_{b} h \eta_{b} \rho
$$

is the mass of the bottom tube, $m_{p}$ is the mass of the primary $\left(\sim 150 \times 10^{3} \mathrm{~kg}\right.$ for a 30 -m-diameter, $50-\mathrm{mm}$ thick segmented mirror $\left.{ }^{8}\right)$, and

$$
m_{c}=\pi\left(D_{1} / 2\right)^{2} t \eta_{c} \rho
$$

is the mass of the mirror cell; $\eta_{t}, \eta_{b}$, and $\eta_{c}$ are space-frame filling factors, and $\rho$ is the density of the space-frame material. $h$ is the distance of the elevation axis in front of the primary. It is the key variable in this analysis. A constant secondarymirror aspect ratio gives $m_{s} \propto D_{2}{ }^{3}$, whereas a particular primary segment size, and hence thickness, gives $m_{p} \propto D_{1}{ }^{2}$.

For matched gravitational deflections perpendicular to the optical axis,

$$
\frac{m_{s}}{k_{t}}+\frac{3 m_{t}}{8 k_{t}}=\frac{3 m_{b}}{8 k_{b}}+\frac{m_{p}}{k_{b}}+\frac{m_{c}}{k_{b}},
$$

where

$$
\begin{aligned}
& k_{t}=\left(\frac{\eta_{t}}{3}\right) \frac{3 \pi E D_{1}^{3} a_{t}}{8(d-h)^{3}}, \\
& k_{b}=\left(\frac{\eta_{b}}{3}\right) \frac{3 \pi E D_{1}^{3} a_{b}}{8 h^{3}},
\end{aligned}
$$

respectively, are the top and bottom tube stiffnesses for end loading (see Appendix A) and the stiffnesses for uniform loading are a factor of $8 / 3$ higher; $E$ is Young's modulus for the tube material. Given the tube lengths and space-frame filling factors, Eqs. (1) and (5) can be solved for the tube wall thicknesses. To match gravitational deflections along the optical axis, we must also adjust the relative axial stiffnesses of the top and bottom tubes but without significantly changing the relative radial stiffnesses. We can do this by adding beams parallel to the optical axis or by changing the axial stiffness of the spider arms in the secondary support.

\section{B. Dynamics}

The fundamental mode of the telescope structure in Fig. 1 is a rocking motion roughly about the elevation axis. This involves bending of the forks, rigid-body rotation of the entire tube assembly, and bending of the top and bottom tube sections. An equivalent lumped-element, coupled-oscillator model is shown in Fig. 2. The forks, the azimuth drives, and the elevation drives are represented by a single spring with stiffness

$$
k_{1}=\left(\frac{2}{k_{d}}+\frac{1}{k_{f}}\right)^{-1},
$$




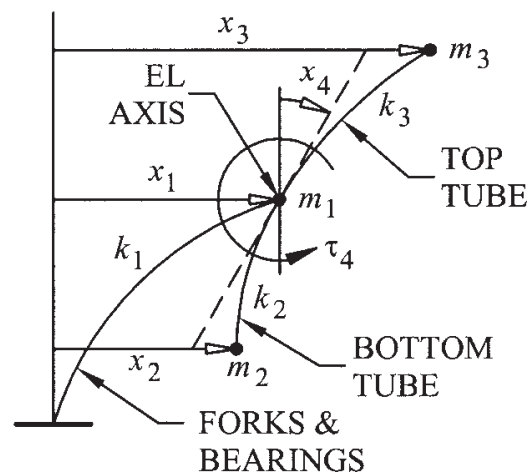

Fig. 2. Lumped-element, coupled-oscillator model of the telescope in Fig. 1: $x_{1}, x_{2}$, and $x_{3}$, lateral displacements of the forks and of the bottom and the top tubes respectively; $x_{4}$, rotation of the entire tube about the elevation axis; $k$, spring constant (e.g., in units of $\mathrm{N}$

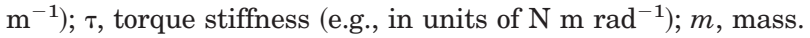

where $k_{d}$ is the stiffness of a drive and

$$
k_{f}=\left(\frac{\eta_{f}}{3}\right) \frac{9 E D_{1}^{4}}{64 L^{3}}
$$

Rigid-body rotation of the tube (through angle $x_{4}$ ) is resisted by elevation-axis torque stiffness $\tau_{4}=k_{d} r^{2}$

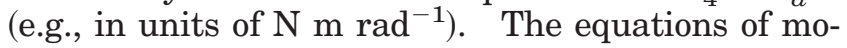
tion for Fig. 2 are

$$
\begin{aligned}
0= & m_{1} \ddot{x}_{1}+k_{1} x_{1}+k_{2}\left(h x_{4}-x_{1}+x_{2}\right)+k_{3}\left[x_{3}-x_{1}\right. \\
& \left.-(d-h) x_{4}\right], \\
0= & m_{2} \ddot{x}_{2}+k_{2}\left(h x_{4}-x_{1}+x_{2}\right)-\tau_{4} x_{4} / h, \\
0= & m_{3} \ddot{x}_{3}+k_{3}\left[x_{3}-x_{1}-(d-h) x_{4}\right]+\tau_{4} x_{4} /(d-h), \\
0= & J \ddot{x}_{4}+\tau_{4} x_{4}+k_{3}\left[x_{3}-x_{1}-(d-h) x_{4}\right](d-h) \\
& -k_{2}\left(h x_{4}-x_{1}+x_{2}\right) h
\end{aligned}
$$

where

$$
\begin{aligned}
J= & m_{s}(d-h)^{2}+m_{t} \frac{(d-h)^{2}}{3}+m_{b} \frac{h^{2}}{3}+m_{p} h^{2} \\
& +m_{c}\left(h+\frac{t}{2}\right)^{2}
\end{aligned}
$$

is the moment of inertia of the tube assembly. For a mode involving the entire structure at frequency $\omega$, the solution has the form $x_{i}=q_{i} \cos \omega t$, so Eqs. (15) become

$$
0=\left[\begin{array}{cccc}
k_{1}-k_{2}-k_{3}-\omega^{2} m_{1} & k_{2} & k_{3} & k_{2} h-k_{3}(d-h) \\
-\omega^{2} m_{2} & k_{2}-\omega^{2} m_{2} & 0 & k_{2} h-\tau_{4} / d_{2} \\
-\omega^{2} m_{3} & 0 & k_{3}-\omega^{2} m_{3} & -k_{3}(d-h)+\tau_{4} /(d-h) \\
0 & -k_{2} h & k_{3}(d-h) & \tau_{4}-k_{2} h^{2}-k_{3}(d-h)^{2}-J \omega^{2}
\end{array}\right] \mathbf{q}=\mathbf{A q} .
$$

is the stiffness of the forks for uniform loading (see Appendix A). Equation (9) models the forks as a single space-frame beam, $3 D_{1} / 4 \times D_{1} / 2 \times L$, where

$$
L=\left[(h+t)^{2}+\left(D_{1} / 2\right)^{2}\right]^{1 / 2} .
$$

The mass of the forks (represented by $m_{1}$ in Fig. 2) is

$$
m_{f}=\frac{3 D_{1}^{2} L \eta_{f} \rho}{8} .
$$

The top and bottom tube models are not straightforward because part of the mass is distributed, but effective stiffnesses for the low-order modes can be estimated from gravitational deflections of the tube with the telescope pointing at the horizon [Eq. (5)]. For the bottom tube,

$$
\begin{aligned}
k_{2} & \approx k_{b} \frac{\left(m_{p}+m_{c}+m_{b}\right)}{\left(m_{p}+m_{c}+3 m_{b} / 8\right)}, \\
m_{2} & =m_{b}+m_{p}+m_{c} ;
\end{aligned}
$$

for the top tube,

$$
\begin{aligned}
& k_{3} \approx k_{t} \frac{\left(m_{s}+m_{t}\right)}{\left(m_{s}+3 m_{t} / 8\right)}, \\
& m_{3}=m_{t}+m_{s} .
\end{aligned}
$$

The smallest positive nonzero $\operatorname{root}$ of $\operatorname{det}(\mathbf{A})$ is the fundamental frequency of the telescope. It can be found numerically after some tedious algebra to develop the 8th-order polynomial corresponding to $\operatorname{det}(\mathbf{A})$.

\section{Blockage and Decenter}

The fraction of the primary that is blocked by the secondary and the spider is

$$
\zeta=\left(\frac{D_{2}}{D_{1}}\right)^{2}+\frac{8 w}{\pi D_{1}^{2}}\left(D_{1}-D_{2}\right),
$$

where the first term is the blockage that is due to the secondary mirror itself and the second term is the blockage for a spider with four arms, each of width $w$. (For the Keck telescopes, $w=25 \mathrm{~mm}, D_{1}=10 \mathrm{~m}$, and $D_{2}=1.5 \mathrm{~m}$, so the secondary and spider blockages are $\sim 2 \%$ and $0.5 \%$ respectively. ${ }^{1)}$ The diameter of the secondary is ${ }^{9}$

$$
D_{2}=\frac{(d+e) D_{1}}{f}+2 \alpha d,
$$

where $e$ is the back focal distance $\left(e=D_{1} / 2-h\right.$ if the Nasmyth focus is at the tube wall), $f$ is the final focal length, $\alpha$ is the angular radius of the field of view, $d=$ $(f-e) /(\gamma+1)$ is the primary to secondary spacing, 

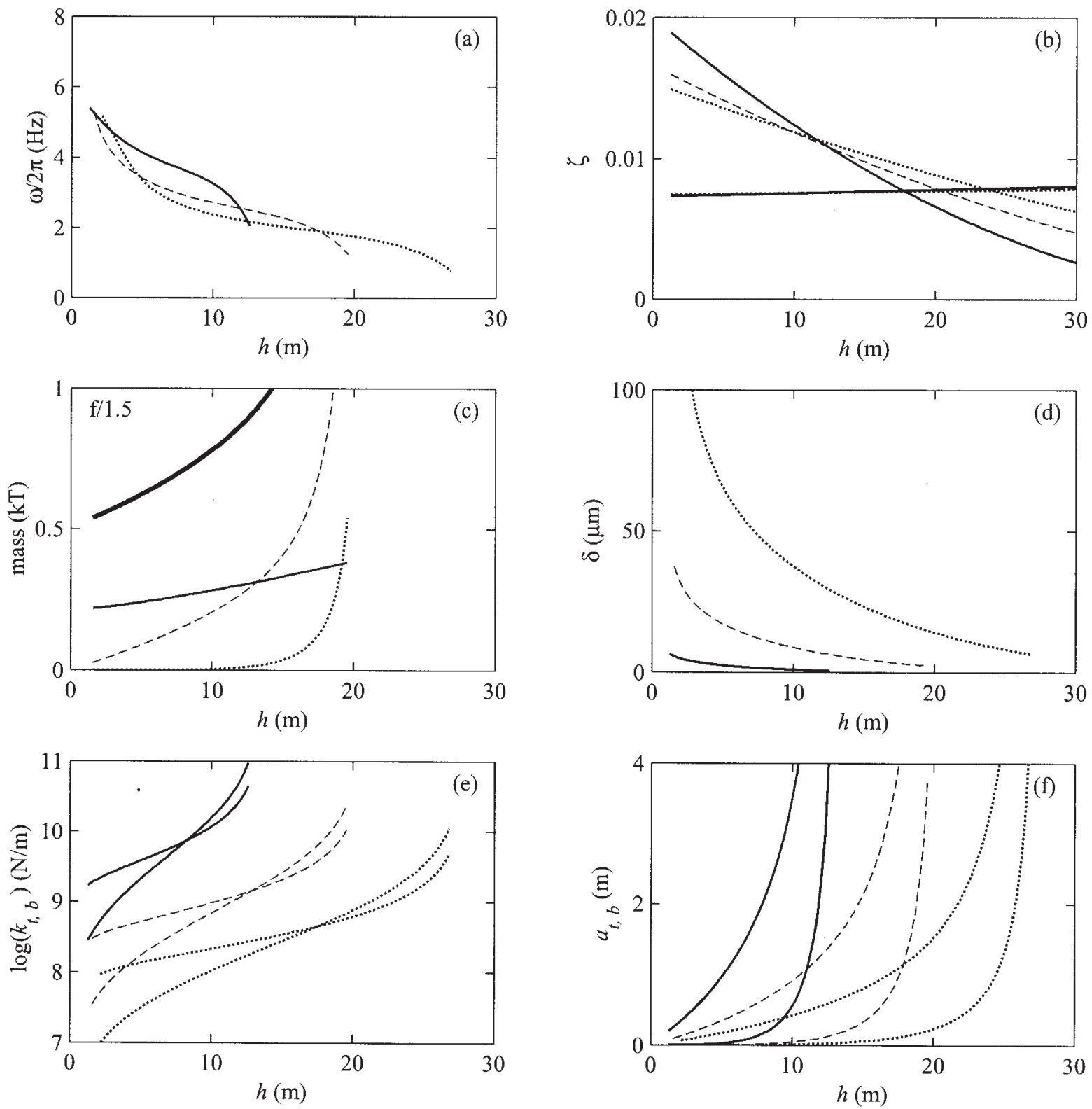

Fig. 3. (a) Fundamental frequency, (b) blockage, (c) mass contributions, and (d) secondary decenter in a 5 -m $\mathrm{s}^{-1}$ wind; (e) tube stiffness for end loading and (f) tube wall thickness for a 30-m telescope as in Fig. $1 ; h$, distance of the elevation axis in front of the primary. In all the figures except (c) the curves represent different primary mirror focal ratios: $f / 1$ (solid curves), $f / 1.5$ (dashed curves), and $f / 2$ (dotted curves). The mass contributions in (c) are $m_{f}$ (thinner solid curve), $m_{t}$ (dashed curve), $m_{b}$ (dotted curve), and total mass (thicker solid curve), all for an $f / 1.5$ primary. In (e) the upper curve near $h=0$ is for the bottom tube, and in (f) the upper curve is for the top tube. Blockages in (b) are for the spider (roughly horizontal curves) and the secondary mirror (curves running from top left to bottom right). Telescope parameters are given in Table 1.

$\gamma=f / f_{1}$ is the secondary magnification, and $f_{1}$ is the primary focal length.

Wind buffeting causes bending and rigid-body motion of the tube. The corresponding image motion can be corrected by a fast-steering mirror (e.g., the tertiary), but a decenter of the secondary causes higher-order aberrations. ${ }^{10}$ It is difficult to estimate the decenter because the spectrum and the correlation length of wind pressure fluctuations vary over the telescope, but a useful upper bound can be obtained from the static deflection of the top tube in a uniform air flow. A space-frame tube with $n$ sides typically has $2 n$ roughly axial members and at least one ring (see Appendix A), so for a hexagonal tube the wind cross section is

$$
A_{t} \sim 12 a_{t}(d-h)+\pi D_{1} a_{t}
$$

The cross section of the secondary mirror is

$$
A_{s} \sim D_{2}^{2} / \beta,
$$


Table 1. Telescope Parameters for Figs. 3 and 6 (below)

\begin{tabular}{|c|c|c|c|}
\hline Parameter & Value & Unit & Description \\
\hline$D_{1}$ & 30 & $\mathrm{~m}$ & Primary diameter \\
\hline$\gamma$ & 10 & & Secondary magnification \\
\hline$e$ & $D_{1} / 2-h$ & & Back focal distance \\
\hline$\alpha$ & 10 & $\operatorname{arcmin}$ & Radius of field of view \\
\hline$t$ & $D_{1} / 6$ & & Primary mirror cell thickness \\
\hline$R$ & $D_{1} / 2$ & & c-ring radius \\
\hline$r$ & 2 & $\mathrm{~m}$ & Radius of elevation drive ring \\
\hline$m_{s}$ & $10^{4}\left(D_{2} / 4\right)^{3}$ & $\mathrm{~kg}$ & Secondary mass $\left(D_{2}\right.$ in meters $)$ \\
\hline$\beta$ & 6 & & Secondary aspect ratio \\
\hline$m_{p}$ & $150 \times 10^{3}\left(D_{1} / 30\right)^{2}$ & $\mathrm{~kg}$ & Primary mass ( $D_{1}$ in meters) \\
\hline$m_{w}$ & 0 & $\mathrm{~kg}$ & Counterweight mass \\
\hline$\eta_{t}, \eta_{b}$ & 0.01 & & Tube filling factors \\
\hline$\eta_{f}, \eta_{c}, \eta_{x}, \eta_{r}$ & 0.005 & & Space-frame filling factors \\
\hline$k_{d}$ & $10^{10}$ & $\mathrm{~N} \mathrm{~m}^{-1}$ & Drive stiffness \\
\hline$w$ & 0.1 & $\mathrm{~m}$ & Spider arm width \\
\hline$v$ & 5 & $\mathrm{~m} \mathrm{~s}^{-1}$ & Wind speed \\
\hline$\rho_{\text {air }}$ & 1.29 & $\mathrm{~kg} \mathrm{~m}^{-3}$ & Density of air \\
\hline$E$ & $2 \times 10^{11}$ & $\mathrm{~Pa}$ & Young's modulus for steel \\
\hline$Y$ & $2 \times 10^{8}$ & $\mathrm{~Pa}$ & Yield modulus for steel \\
\hline$\rho$ & 7833 & $\mathrm{~kg} \mathrm{~m} \mathrm{~m}^{-3}$ & Density of steel \\
\hline$S$ & 2 & & Stress safety factor \\
\hline
\end{tabular}

where $\beta$ is the aspect ratio, and the cross section of a four-arm spider is

$$
A_{x} \sim 2 w\left(D_{1}-D_{2}\right)\left(D_{2} / \beta\right) .
$$

If the air flow has a high, but subcritical, Reynolds number, the drag coefficient of the structure is roughly 1 , and the decenter of the secondary is

$$
\delta \sim \frac{1}{2} \rho_{\text {air }} v^{2}\left(\frac{3}{8} A_{t}+A_{s}+A_{x}\right) \frac{1}{k_{t}},
$$

where $\rho_{\text {air }}$ is the density of air and $v$ is the wind speed. ${ }^{11}$ The factor $3 / 8$ represents the higher stiffness for uniform wind loading on the tube walls compared with end loading that is the result of wind forces on the secondary and spider.

\section{Results}

Figure 3 shows the fundamental frequency, blockage, and wind-induced secondary decenter for a $30-\mathrm{m}$ tube-style telescope with the parameters given in Table 1. The space-frame filling factors in Table 1 are from the California Extremely Large Telescope conceptual design, ${ }^{8}$ and the drive stiffness is from a $30-\mathrm{m}$ telescope design based on the Large Binocular Telescope. ${ }^{12}$ The drive stiffness and the diameter of the elevation drive ring are important for setting the frequency scale in Fig. 3(a). A large drive ring gives high torque stiffness and high fundamental frequency, but there is a severe mass penalty because the ring must have a filling factor of at least 0.1; otherwise its stiffness will limit $\tau_{4}$. For Fig. $3, r=2$ $\mathrm{m}$, which was scaled from the Gemini elevation trunnion design, ${ }^{5}$ but a larger drive ring with a high filling factor may be possible. As the elevation axis is moved toward the secondary, the fundamental fre- quency decreases because the mass of the tube increases. At $h=d / 2$, it becomes impossible to balance the tube; the mass explodes and the fundamental frequency of the telescope drops to zero. At small $h$, the fundamental frequency curves cross because the motion of the tube changes from rigid-body rotation to bending. Stress and buckling in the tube members limit the tube-wall thickness (Section 3), so designs with $h$ close to zero may not be practical; they are in any case of limited interest because the tube stiffness is low, so wind-induced decenter of the secondary is high. If $h$ is small, the bottom tube will be stiff enough to support the elevation axis, as in the Gemini design,5; otherwise a stiff ring must be included between the top and bottom tubes, as in the Keck telescopes. ${ }^{1}$ Adding a ring will increase the mass of the telescope and reduce its fundamental frequency.

\section{Telescope with a c-Ring Mount and a Quadrupod}

\section{A. Structure}

Figure 4 shows a telescope with a quadrupod secondary support and c-rings that support the back of the primary. The c-rings provide the elevation axis bearing surfaces, and the azimuth bearings run on a track on a stiff pier, as in the fork mount of Fig. 1. If the c-rings are large, the elevation axis can be in front of the primary, and the structure can be balanced without a counterweight. (The Lovell Telescope, ${ }^{13}$ for example, uses this approach.) Active control of the secondary position is required for maintaining the optical alignment of the telescope, so the quadrupod can be designed to maximize the fundamental frequency or to limit wind-induced decenter of the secondary. For the following analysis, the design constraints are a balanced structure and minimum 


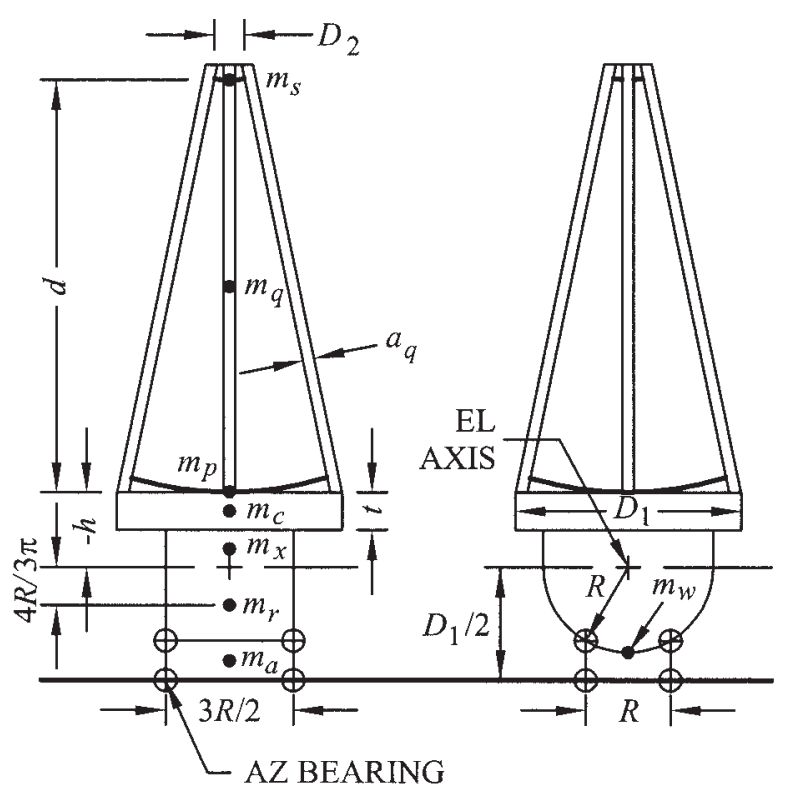

Fig. 4. Telescope with a quadrupod and a c-ring mount.

blockage, i.e., minimum quadrupod mass. The second constraint, which maximizes the fundamental frequency of the telescope, is a good choice because it also maximizes the control bandwidth for pointing and optical alignment.

Stress in a horizontal quadrupod leg sets a lower limit on the leg's diameter. The stress is minimized if the leg is as thick as possible in the direction of the gravity vector, so a quadrupod leg in the plane of the elevation axis should have an aspect ratio of 1 . A leg in a different position can have a higher aspect ratio to reduce the stress without compromising the blockage. The following analysis is for a simple quadrupod with legs made from round tubes. The maximum stress in a horizontal leg supported at each end is ${ }^{14}$

$$
\sigma=\frac{m_{\operatorname{leg} g} g l}{8 Z}
$$

where

$$
m_{\text {leg }}=\frac{\pi a_{q}^{2} \eta_{q} l \rho}{4}
$$

is the mass of the leg,

$$
l=\left(\frac{D_{1}^{2}}{4}+d^{2}\right)^{1 / 2}
$$

is the length of the leg, and

$$
Z=\frac{\pi}{32} a_{q}^{3} \eta_{q}\left(2-\eta_{q}\right)
$$

is the section modulus; $\eta_{q}$ is the leg filling factor, and $g$ is the acceleration that is due to gravity. The stress must be smaller than the yield modulus $Y$ by a safety factor $S$, so the minimum leg diameter is

$$
a_{q}>\frac{S g \rho l^{2}}{Y\left(2-\eta_{q}\right)} .
$$

As an aside, the Euler bucking load for a vertical leg is

$$
F_{\text {cr }}=\frac{\pi^{2} E I}{l^{2}},
$$

where

$$
I=\frac{\pi}{64} a_{q}^{4} \eta_{q}\left(2-\eta_{q}\right)
$$

is the moment of inertia. ${ }^{14}$ If $m_{\text {leg }} g<F_{\text {cr }} / S$, then

$$
a_{q}^{2}>\frac{16 S g \rho l^{3}}{\pi^{2} E\left(2-\eta_{q}\right)},
$$

which is a less severe constraint than Eq. (28) for steel legs longer than $\sim 4 \mathrm{~m}$ if $S=2$.

Refer to Fig. 4; for a balanced telescope,

$$
\begin{aligned}
& m_{s}(d-h)+m_{q}\left(\frac{d}{2}-h\right)-m_{p} h \\
& -m_{c}\left(h+\frac{t}{2}\right)-m_{x} \frac{(h+t)}{2} \\
& =m_{r} \frac{4 R}{3 \pi}+m_{w} R,
\end{aligned}
$$

where

$$
m_{q}=4 m_{\text {leg }}=\pi a_{q}{ }^{2} \eta_{q} l_{\rho}
$$

is the mass of the quadrupod,

$$
m_{x} \approx 3 R^{2} \rho \eta_{x}(-h-t)
$$

is the mass of the box that couples the mirror cell to the c-rings,

$$
m_{r}=\frac{3}{4} \pi R^{3} \rho \eta_{r}
$$

is the mass of the c-rings, and $m_{w}$ is the mass of the counterweight; $m_{s}, m_{p}$, and $m_{c}$ are the same as for Fig. 1. For $h>-t, m_{x}$ represents the region where the mirror cell overlaps the c-rings, so modeling $m_{x}$ as a simple rectangular box, $R \times 3 R / 2 \times(t-h)$, causes an error in Eq. (32) that increases with $h$. Given dimensions and space-frame filling factors for the mirror cell and the c-ring assembly, and a stress safety factor, inequality (28) and Eq. (32) can be solved for $a_{q}$ and $\eta_{q}$.

\section{B. Dynamics}

The fundamental mode of the telescope is again a rocking motion, roughly about the elevation axis. In this case the telescope can be broken up into a simple series-connected array of springs and masses, as in 
Fig. 5. The azimuth and elevation drives, azimuth platform, c-rings, and the box that connects the c-rings to the mirror cell are all modeled as a single beam, $R \times 3 R / 2 \times D_{1} / 2-h-t$, with stiffness

$\tau_{1}=\left[\frac{2}{k_{d}}+\frac{3}{R^{4} E \eta_{r}}\left(\frac{D_{1}}{2}-h-t\right)^{3}\right]^{-1} \frac{1}{2}\left(\frac{D_{1}}{2}-h-t\right)^{2}$.

(The torque stiffness of a uniformly loaded beam of length $d$ is $\tau=k d^{2} / 2$, e.g., in units of $\mathrm{N} \mathrm{m} \mathrm{rad}{ }^{-1}$, where $k$ is the beam stiffness for uniform loading, e.g., in units of $\mathrm{N} \mathrm{m}^{-1}$.) The moment of inertia of the beam is

$$
\begin{aligned}
J_{1} \approx & \frac{3 R}{2}\left[\frac{R}{3}\left(\frac{D_{1}}{2}-\frac{\sqrt{3} R}{2}\right)^{3}+\frac{\pi R^{2}}{2}\left(\frac{D_{1}}{2}-\frac{4 R}{3 \pi}\right)^{2}\right. \\
& \left.-2 R(h+t)\left(\frac{D_{1}}{2}-\frac{h+t}{2}\right)^{2}\right] \rho \eta_{r} \\
& +m_{w}\left(\frac{D_{1}}{2}-R\right)^{2}
\end{aligned}
$$

The stiffness of the mirror cell, or some similar structure that supports the quadrupod, can be estimated from the stiffness of a uniformly loaded circular plate supported mainly near the center. This is roughly equivalent to a simply supported plate, for which the axial stiffness is ${ }^{15}$

$$
k_{c}=\left(\frac{\eta_{c}}{3}\right) \frac{16 \pi E t^{3}}{3(1-v)(5+v)\left(D_{1} / 2\right)^{2}},
$$

where $v$ is Poisson's ratio for the plate material. The stiffness of a plate scales as the inverse square of a mode feature size, so the tilt stiffness is roughly a factor 4 smaller than the axial stiffness. Hence,

$$
\tau_{2} \sim \frac{1}{4} k_{c} \frac{D_{1}^{2}}{2}=\left(\frac{\eta_{c}}{3}\right) \frac{8 \pi E t^{3}}{3(1-v)(5+v)} .
$$

For steel, $v \approx 0.3$, so

$$
\tau_{2} \sim\left(\frac{\eta_{c}}{3}\right) 2 E t^{3} .
$$

We can obtain a similar result by modeling the cell as a pair of rectangular beams, each with section $t \times$ $D_{1} / 2$ and length $D_{1} / 2$. The moment of inertia of the cell and the primary mirror is

$$
J_{2}=\left(m_{p}+m_{c}\right)\left(\frac{D_{1}}{4}\right)^{2} .
$$

For the quadrupod, the stiffness for end loading is

$$
k_{q} \approx \frac{3}{8} \frac{\pi E D_{1}^{2} a_{q}^{2} \eta_{q}}{2 d^{3}}
$$

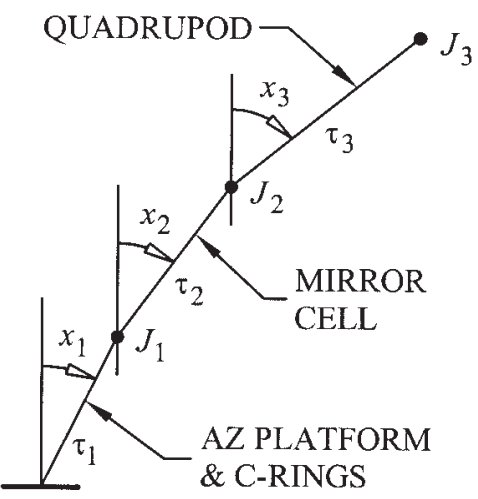

Fig. 5. Lumped-element, coupled-oscillator model of the telescope in Fig. 5: $x$, angular displacement; $\tau$, torque stiffness (e.g., in

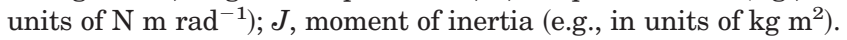

(see Appendix A), so

$$
\tau_{3}=\frac{8}{3} k_{q} \frac{d^{2}}{2} \approx \frac{\pi E D_{1}^{2} a_{q}^{2} \eta_{q}}{4 d} .
$$

The moment of inertia is

$$
J_{3}=\frac{m_{q} d^{2}}{3}+m_{s} d^{2}
$$

The equations of motion for Fig. 5 are

$$
\begin{aligned}
& 0=J_{1} \ddot{x}_{1}+\tau_{1} x_{1}+\tau_{2}\left(x_{2}-x_{1}\right), \\
& 0=J_{2} \ddot{x}_{2}+\tau_{2}\left(x_{2}-x_{1}\right)-\tau_{3}\left(x_{3}-x_{2}\right), \\
& 0=J_{3} \ddot{x}_{3}+\tau_{3}\left(x_{3}-x_{2}\right),
\end{aligned}
$$

and, for a solution of the form $x_{i}=q_{i} \cos \omega t$,

$$
\begin{aligned}
0 & =\left[\begin{array}{ccc}
\tau_{1}+\tau_{2}-\omega^{2} J_{1} & -\tau_{2} & 0 \\
-\tau_{2} & \tau_{2}+\tau_{3}-\omega^{2} J_{2} & -\tau_{3} \\
0 & -\tau_{3} & k_{3}-\omega^{2} J_{3}
\end{array}\right] \mathbf{q} \\
& =\mathbf{A q} .
\end{aligned}
$$

The fundamental frequency is again the smallest positive root of $\operatorname{det}(\mathrm{A})$.

C. Blockage and Decenter

The blockage for the telescope in Fig. 4 is

$$
\zeta=\left(\frac{D_{2}}{D_{1}}\right)^{2}+\frac{8 a_{q}}{\pi D_{1}^{2}}\left(D_{1}-D_{2}\right),
$$

and the decenter of the secondary that is due to uniform wind loading is

$$
\delta \sim \frac{1}{2} \rho_{\text {air }} v^{2}\left(\frac{3}{8} A_{q}+A_{s}\right) \frac{1}{k_{q}},
$$

where

$$
A_{q}=4 a_{q} l
$$

is the cross section of the quadrupod legs and $A_{s}$ is the cross section of the secondary [Eq. (21)]. 

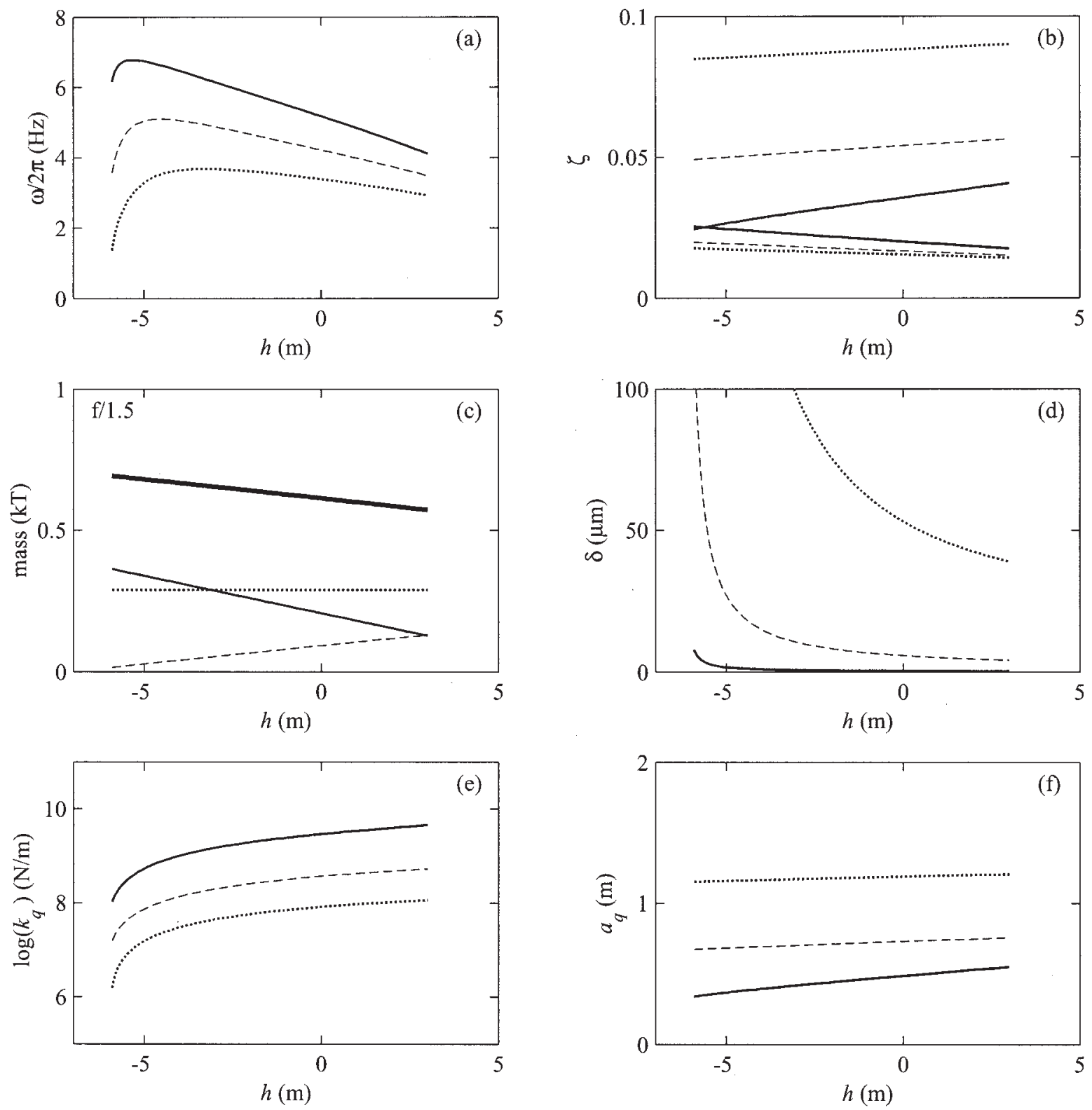

Fig. 6. (a) Fundamental frequency, (b) blockage, (c) mass contributions, and (d) secondary decenter in a 5-m $\mathrm{s}^{-1}$ wind; (e) quadrupod stiffness for uniform loading and (f) quadrupod tube diameter for a 30-m telescope as in Fig. 5 , with c-rings of diameter $D_{1} ; h$, distance of the elevation axis in front of the primary. In all the figures except (c) the curves represent different primary mirror focal ratios: $f / 1$ (solid curves), $f / 1.5$ (dashed curves), and $f / 2$ (dotted curves). The mass contributions in (c) are $m_{w}+m_{r}+m_{x}$ (thinner solid curve), $m_{q}$ (dashed curve), $m_{c}+m_{p}$ (dotted curve), and total mass (thicker solid curve), all for an $f / 1.5$ primary. Blockages in (b) are for the secondary mirror (lower curves) and the quadrupod legs (upper curves). Telescope parameters are given in Table 1.

\section{Results}

Figure 6 shows the fundamental frequency, blockage, and wind-induced secondary decenter for a $30-\mathrm{m}$ quadrupod-style telescope with the parameters listed in Table 1. High fundamental frequency is again associated with low secondary support mass, which in this case means moving the elevation axis further behind the primary, consistent with most existing radio telescope designs. If $h$ is made too negative, the dynamics of the telescope are dominated by the secondary oscillating on a floppy quadrupod, and the fundamental frequency curves turn over. The fun- damental frequencies in Fig. 6(a) [and also in Fig. $3(\mathrm{a})]$ are too high by a factor of $\sim \sqrt{2}$ because the simple space-frame models tend to overestimate stiffnesses by a factor of $\sim 2$ (see Appendix A). A real $30-\mathrm{m} f / 1$ telescope might achieve a fundamental frequency of $\sim 4.8 \mathrm{~Hz}$.

The blockage in Fig. 6(b) is dominated by the quadrupod legs and decreases with quadrupod mass, so blockage and fundamental frequency both work to push the elevation axis away from the secondary. This situation is quite different from that for a tubestyle telescope, in which the blockage is dominated by 


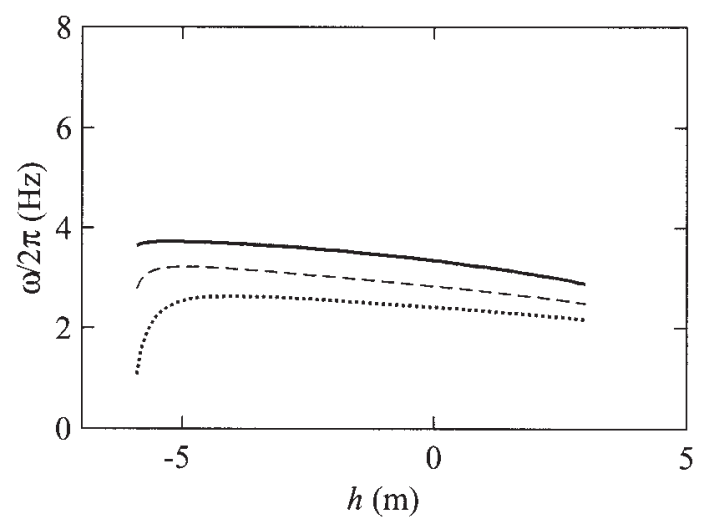

Fig. 7. Same as Fig. 4 (a) but with $R=D_{1} / 4$ and $m_{w}=245 \times 10^{3}$ $\mathrm{kg}$.

the secondary mirror [see Fig. 3(b)]. We could reduce the quadrupod blockage by changing to a twotier support with a lower space-frame tube and an upper multipod with fairly thin legs. ${ }^{8}$

If the elevation axis is behind the primary, we can reduce the size of the c-rings without limiting the elevation range of the telescope, which will leave more space for instruments. Figure 7 shows the effect of reducing the c-ring diameter by a factor of 2 and adding a counterweight to balance the telescope. The fundamental frequency is lower than in Fig. 6(a) because the counterweight adds mass, which increases the moment of inertia of the c-rings, but it contributes nothing to the stiffness of the structure. We can at least partly recover the performance by increasing the filling factor of the c-ring space frames and reducing the mass of the counterweight. This configuration is quite attractive because a high spaceframe filling factor has the important practical advantage of high stiffness on small spatial scales.

With a fast primary, and with the elevation axis positioned for high fundamental frequency, the quadrupod and tube-style telescope designs have similar fundamental frequency, blockage, wind-induced secondary decenter, and total mass.

\section{Wind Buffeting}

The analysis of Sections 2 and 3 allows us to compare the effect of wind buffeting on quadrupod and tube structures with the same total mass. From Eqs. (26) and (33) and relation (42), the stiffness of a quadrupod of height $d \gg D_{1} / 2$ and mass $m$ is

$$
k_{q} \approx \frac{3 E D_{1}^{2} m}{16 \rho d^{4}}
$$

so the ratio of leg cross section [Eq. (49)] to stiffness is

$$
\Delta_{q} \sim \frac{64 a_{q} \rho d^{5}}{3 E D_{1}^{2} m}
$$

The wind-induced decenter of the secondary is proportional to $\Delta$. From Eqs. (2) and (6), the stiffness of a tube of length $d$ and mass $m$ is

$$
k_{t}=\frac{E D_{1}^{2} m}{8 \rho d^{4}}
$$

which is smaller than $k_{q}$ by a factor of $3 / 2$. For a hexagonal tube, the cross section of the axial members is $\sim 12 a_{t} d$ [see Eq. (20)], so the ratio of cross section to stiffness is

$$
\Delta_{t} \sim \frac{96 a_{t} \rho d^{5}}{E D_{1}^{2} m} .
$$

Hence,

$$
\frac{\Delta_{t}}{\Delta_{q}} \sim \frac{9 a_{t}}{2 a_{q}} .
$$

The stress constraint of inequality (28) gives a lower limit for the diameter of the quadrupod legs, and this also applies to members that run the full length of a tube. Thus a quadrupod with the minimum leg diameter (i.e., minimum blockage) will have at least a $9 / 2$ times lower decenter than a tube of the same total mass. If we include the cross sections of the spider and the top tube ring, the advantage of the quadrupod is even greater. The better performance of the quadrupod is not obvious from Figs. 3(d) and $6(d)$ because the design constraints for the two telescopes are different. The quadrupod was designed for minimum blockage, i.e., minimum mass, but the tube was designed to maintain alignment of the optics. The result is in a fairly massive top tube, which has roughly the same decenter as the quadrupod.

\section{Conclusions}

The new results from this study are that

1. For a fast 30-m telescope with a c-ring mount and a quadrupod, the optimum location for the elevation axis is behind the primary. Wind-induced decenter of the secondary increases rapidly with primary focal ratio, and with an $f / 2$ primary the decenter is large enough to favor an elevation axis just in front of the primary.

2. The penalty for moving the elevation axis in front of the primary in a c-ring mount is a $20 \%$ (for $f / 2$ ) to $40 \%$ (for $f / 1$ ) decrease in the fundamental frequency of the telescope. This decrease will reduce the control bandwidth for pointing and optical alignment, but the fundamental frequency will still be above most of the power in wind disturbances. The result is important because it shows that we can move the elevation axis, e.g., to provide access to multiple Nasmyth instruments, without seriously compromising the telescope control.

\section{Appendix A: Stiffness Models}

Here, simple beam and finite-element models of the stiffness of space-frame structures are compared. In 

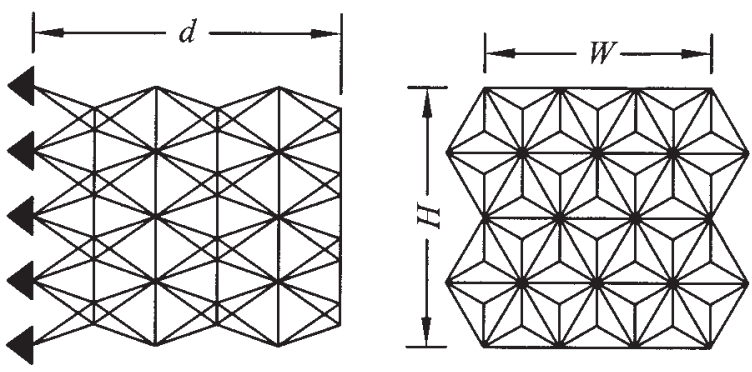

Fig. 8. Space-frame beam made from layers of tetrahedrons and sheets of hexagons. Filled triangles, fixed points for FEA.

a space frame, roughly one third of the members run across the structure in each of three orthogonal directions. The stiffness should therefore be $\sim \eta / 3$ times the stiffness of the corresponding solid, where $\eta$ is the space-frame filling factor. For the spaceframe beam in Fig. 8 the expected stiffness for end loading is

$$
k=\frac{\eta}{3} \frac{3 E I}{d^{3}}
$$

where $I=W H^{3} / 12$ is the moment of inertia. ${ }^{14}$ Figure 9 shows finite-element analysis (FEA) results for beams made from steel tubes of several sizes. As expected, $k \propto \eta$, but the simple beam model tends to overestimate the stiffness by a factor of $\sim 3 / 2$. For gravitational (i.e., uniform) loading, the stiffness of the finite-element model increases by 2.44 , which is close to the expected value of $8 / 3$.

The hollow core of a space-frame tube restricts the location of frame members, so we might expect a stiffness penalty. For an ideal tube, with dimensions as in Fig. 10, the expected stiffness for end loading is

$$
k=\frac{\eta}{3} \frac{3 \pi E D^{3} a}{8 d^{3}}
$$

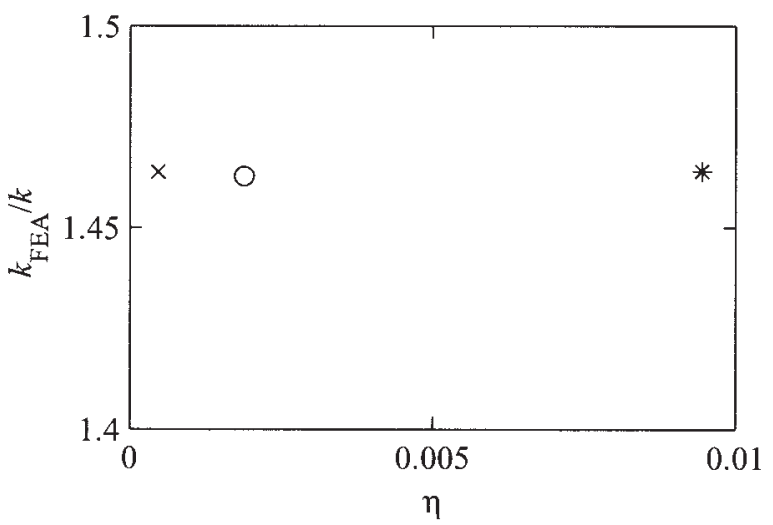

Fig. 9. Stiffness of the space-frame beam in Fig. 8 from FEA, divided by the stiffness predicted by Eq. (A1). The beam dimensions are $W=5 \mathrm{~m}, H=4.6 \mathrm{~m}$, and $d=8.2 \mathrm{~m}$, with 1 -m tetrahedrons; frame members are steel tubes $10 \mathrm{~mm}$ in diameter $\times 1 \mathrm{~mm}$ wall (cross), $20 \mathrm{~mm} \times 2 \mathrm{~mm}$ (circle), and $40 \mathrm{~mm} \times 5 \mathrm{~mm}$ (asterisk).
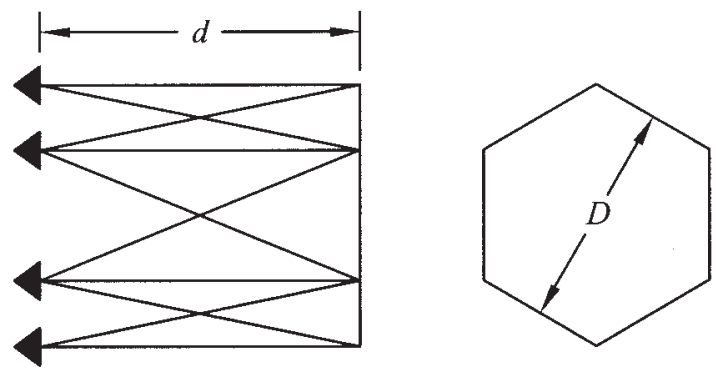

Fig. 10. Space-frame tube.

where $a \ll D$ is the tube-wall thickness. A hexagonal tube with $D=30 \mathrm{~m}$ and $d=40 \mathrm{~m}$, made from 10 -mm-wall steel tubes with $a=1 \mathrm{~m}$, has $\eta=5.2 \times$ $10^{-3}$, so the expected stiffness is $1.7 \times 10^{8} \mathrm{~N} \mathrm{~m}^{-1}$ for end loading or $4.6 \times 10^{8} \mathrm{~N} \mathrm{~m}^{-1}$ for uniform loading. The corresponding FEA results are $1.0 \times 10^{8} \mathrm{~N} \mathrm{~m}^{-1}$ for end loading and $1.9 \times 10^{8} \mathrm{~N} \mathrm{~m}^{-1}$ for gravitational loading; the latter value slightly underestimates the uniform loading stiffness because the gravitational deflection is increased by the weight of the ring at the top of the tube. Equation (A2) overestimates the tube stiffness by a factor of $\sim 2$, so the model is a little worse than that for the dense space-frame beam of Fig. 8.

For a quadrupod as in Fig. 11 we can estimate the deflection, and hence the stiffness, by integrating stress along a beam that has the same moment of inertia as the quadrupod legs. ${ }^{16}$ This approach tends to overestimate the stiffness because it does not account for the bending of individual legs. The moment of inertia of the quadrupod is

$$
I(z) \approx 2 \frac{\pi a^{2} \eta}{4}\left[\left(1-\frac{z}{d}\right) \frac{D}{2}\right]^{2}
$$

where $\eta$ is the filling factor of the tubes. With uniform pressure $Q$, the bending moment is

$$
M(z)=-Q a\left(\frac{d^{2}}{2}-\mathrm{d} z+\frac{z^{2}}{2}\right)
$$

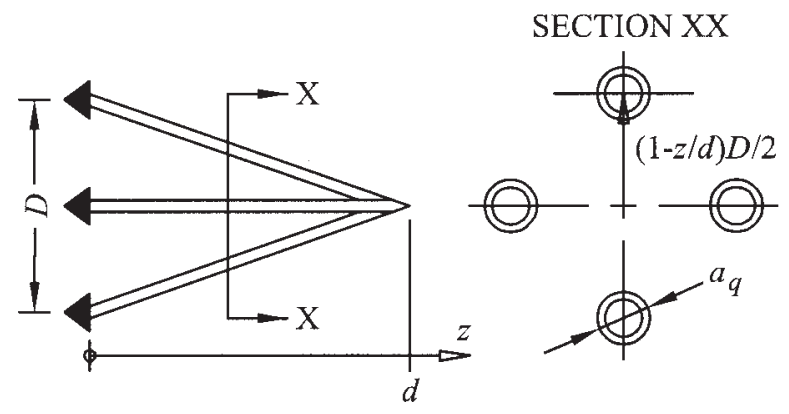

Fig. 11. Quadrupod. 
and the deflection gradient along the quadrupod is

$$
\delta^{\prime}(z)=\int \frac{M(z)}{E I(z)} \mathrm{d} z \approx \frac{4 Q d^{3}}{\pi E D^{2} a \eta}\left(1-\frac{z}{d}\right) .
$$

The deflection at the apex is

$$
\delta=\int_{0}^{d} \delta^{\prime}(z) \mathrm{d} z \approx \frac{2 Q d^{4}}{\pi E D^{2} a \eta},
$$

so the stiffness for end loading is

$$
k=\frac{3}{8} \frac{Q}{\delta} \approx \frac{3}{8} \frac{\pi E D^{2} a^{2} \eta}{2 d^{3}} .
$$

A quadrupod with $D=30 \mathrm{~m}$ and $d=40 \mathrm{~m}$, made from 10-mm-wall steel tubes with $a=1 \mathrm{~m}$, has $\eta=$ $4.0 \times 10^{-2}$, so the expected stiffness is $6.6 \times 10^{7} \mathrm{~N}$ $\mathrm{m}^{-1}$ for end loading or $1.8 \times 10^{8} \mathrm{~N} \mathrm{~m}^{-1}$ for uniform loading. FEA results for this structure are $3.8 \times 10^{7}$ $\mathrm{N} \mathrm{m}^{-1}$ for end loading and $7.5 \times 10^{7} \mathrm{~N} \mathrm{~m}^{-1}$ for gravitational loading. Expression (A7) overestimates the quadrupod stiffness by a factor of $\sim 2$ for end loads and is a little worse for uniform loads because these are coupled only between the legs as end loads at the apex. Adding thin members in tension between the legs would distribute the coupling and improve the stiffness.

The author thanks Doug MacMartin and Larry Stepp for helpful comments. This study was supported by the Caltech Discovery Fund.

\section{References}

1. J. E. Nelson, T. S. Mast, and S. M. Faber, eds., "The design of the Keck Observatory and Telescope," Keck Observatory Rep. 90 (Keck Observatory, Kamuela, Hawaii, 1985).

2. M. Quattri, F. Dimichino, G. Marchiori, and E. Piccinini, "VLT $8 \mathrm{~m}$ unit telescope main structure: design solutions and per- formance calculations," in Advanced Technology Optical Telescopes V, L. M. Stepp, ed., Proc. SPIE 2199, 986-996 (1994).

3. K. Miyawaki, N. Itoh, R. Sugiyama, and M. Sawa, "Mechanical structure for the Subaru Telescope," in Advanced Technology Optical Telescopes V, L. M. Stepp, ed., Proc. SPIE 2199, 754761 (1994).

4. S. M. Gunnels, "Design of the Magellan Project 6.5 meter Telescope: telescope structure and mechanical systems," in Advanced Technology Optical Telescopes V, L. M. Stepp, ed., Proc. SPIE 2199, 414-427 (1994).

5. K. Raybould, P. Gillett, P. Hatton, G. Pentland, M. Sheehan, and M. Warner, "Gemini Telescope structure design," in Advanced Technology Optical Telescopes V, L. M. Stepp, ed., Proc. SPIE 2199, 376-393 (1994).

6. S. Strom, L. M. Stepp, M. Mountain, and B. Gregory, "Giant Segmented Mirror Telescope: a point design based on science drivers," in Future Giant Telescopes, J. R. P. Angel and R. Gilmozzi, eds., Proc. SPIE 4840, 116-128 (2003).

7. T. Andersen, A. L. Ardeberg, J. Beckers, A. Goncharov, M. Owner-Petersen, H. Riewaldt, R. Snel, and D. Walker, "The Euro50 Extremely Large Telescope," in Future Giant Telescopes, J. R. P. Angel and R. Gilmozzi, eds., Proc. SPIE 4840, 214-225 (2003).

8. J. E. Nelson and T. S. Mast, eds., "Conceptual design for a thirty-meter telescope," CELT Rep. 34 (University of California, Santa Cruz, Santa Cruz, Calif., 2002).

9. J. Nelson, T. Mast, and G. Chanan, "Aberration correction in a telescope with a segmented primary," in Active Telescope Systems, F. J. Roddier, ed., Proc. SPIE 1114, 241-257 (1989).

10. D. J. Schroeder, Astronomical Optics (Academic, San Diego, Calif., 2000), p. 132.

11. P. K. Kundu and I. M. Cohen, Fluid Mechanics (Academic, San Diego, Calif., 2002), p. 344.

12. W. B. Davison, N. J. Woolf, and J. R. P. Angel, "Design and analysis of $20 \mathrm{~m}$ track mounted and $30 \mathrm{~m}$ telescopes," in Future Giant Telescopes, J. R. P. Angel and R. Gilmozzi, eds., Proc. SPIE 4840, 533-540 (2003).

13. R. H. Brown and A. C. B. Lovell, The Exploration of Space by Radio (Chapman \& Hall, London, 1957), p. 194.

14. R. J. Roark and W. C. Young, Formulas for Stress and Strain (McGraw-Hill, New York, 1975), pp. 96, 534.

15. S. Timoshenko and S. Woinowsky-Krieger, Theory of Plates and Shells (McGraw-Hill, New York, 1959), p. 57.

16. R. Richards, Solid Mechanics (CRC Press, Boca Raton, Fla., 2001), p. 150. 